\title{
Communication
}

\section{Nanophotonic Heterostructures for Efficient Propulsion and Radiative Cooling of Relativistic Light Sails}

Ognjen Ilic, Cora M. Went, and Harry A Atwater

Nano Lett., Just Accepted Manuscript • Publication Date (Web): 31 Jul 2018

Downloaded from http://pubs.acs.org on July 31, 2018

\section{Just Accepted}

"Just Accepted" manuscripts have been peer-reviewed and accepted for publication. They are posted online prior to technical editing, formatting for publication and author proofing. The American Chemical Society provides "Just Accepted" as a service to the research community to expedite the dissemination of scientific material as soon as possible after acceptance. "Just Accepted" manuscripts appear in full in PDF format accompanied by an HTML abstract. "Just Accepted" manuscripts have been fully peer reviewed, but should not be considered the official version of record. They are citable by the Digital Object Identifier (DOI®). "Just Accepted" is an optional service offered to authors. Therefore, the "Just Accepted" Web site may not include all articles that will be published in the journal. After a manuscript is technically edited and formatted, it will be removed from the "Just Accepted" Web site and published as an ASAP article. Note that technical editing may introduce minor changes to the manuscript text and/or graphics which could affect content, and all legal disclaimers and ethical guidelines that apply to the journal pertain. ACS cannot be held responsible for errors or consequences arising from the use of information contained in these "Just Accepted" manuscripts. 


\title{
Nanophotonic Heterostructures for Efficient Propulsion and Radiative Cooling of Relativistic Light Sails
}

\author{
Ognjen Ilic, Cora M. Went, and Harry A. Atwater* \\ Thomas J. Watson Laboratories of Applied Physics \\ California Institute of Technology \\ Pasadena, CA 91125
}

Light sails propelled by radiation pressure from high-power lasers have the potential to achieve relativistic spaceflight. In order to propel a spacecraft to relativistic speeds, an ultrathin, gramsized, light sail will need to be stably accelerated by lasers with $\sim \mathrm{MW} / \mathrm{cm}^{2}$ intensities operating in the near infrared spectral range. Such a laser-driven sail requires multiband electromagnetic functionality: it must simultaneously exhibit very low absorptivity in the (Doppler-broadened) laser beam spectrum in the near infrared and high emissivity in the mid infrared for efficient radiative cooling. These immense engineering challenges present an opportunity for nanophotonic design. Here, we show that designed thin-film heterostructures could become multifunctional building-block elements of the light sail, due to their ability to achieve substantial reflectivity while maintaining low absorption in the near infrared, significant emissivity in the mid infrared, and a very low mass. For a light sail carrying a payload, we propose a relevant figure of meritthe reflectivity adjusted area density - that can capture the tradeoff between sail mass and reflectivity, independent of other quantities such as the incident beam power, phased array size, or the payload mass. Furthermore, we present designs for effective thermal management via radiative cooling and compare propulsion efficiencies for several candidate materials, using a general approach that could apply to a broad range of high-power laser propulsion problems.

Keywords: laser propulsion, light sail, optical heterostructures, radiative cooling, Breakthrough Starshot

*haa@caltech.edu

\section{INTRODUCTION}

The concept of light-driven cosmic sails dates back to pioneering works of Tsiolkovsky and Tsander who postulated that the pressure of sunlight could be used to propel a spacecraft ${ }^{1,2}$. This category of light sails - known as solar sails - has been the focus of much research ${ }^{2}$, culminating in the launch of the IKAROS solar sail prototype by the Japanese Aerospace Exploration Agency (JAXA) in 2010 ${ }^{3}$. In contrast to solar sails driven by broadband radiation of relatively weak (e.g., solar) intensity, the concept of laser-propelled light sails envisions illumination by a focused, high-power laser phased array ${ }^{4-7}$. More 
recently, the Breakthrough Starshot Initiative was founded around the idea of using an Earth-based laser source to accelerate an ultralight spacecraft to the relativistic velocities needed to reach the nearest known potentially habitable exoplanet—Proxima Centauri b-in a matter of decades ${ }^{8}$.

In order to propel a spacecraft to relativistic speeds, an ultrathin, gram-sized light sail must be stably accelerated under $\mathrm{MW} / \mathrm{cm}^{2}$ laser intensities, presenting an immense scientific and engineering challenge. Among the fundamental challenges to be addressed is achieving efficient photon momentum transfer while ensuring thermal stability of the light sail under intense laser illumination. Suitable material candidates include dielectrics with low absorption coefficients ${ }^{7,9-12}$, but key insight into photonic designs that can balance the tradeoff between the optical response, mass, and equilibrium temperature of a highspeed sail is missing from the literature.

In this work, we show that designed nanophotonic structures can simultaneously facilitate efficient propulsion and radiative thermal management of a meter-scale light sail. By combining materials with high refractive indices in the near infrared and materials with vibrational modes in the mid infrared, we show that designed thin-film heterostructures can both radiatively cool and have substantial reflectivity for effective momentum transfer. We propose a new figure of merit - termed reflectivity adjusted area density - that can fully capture the trade-off between the reflectivity and the mass of a light sail carrying a payload. As one example, we show that layered thin-film heterostructures of silicon and silica could be designed to operate below a desired equilibrium temperature for a range of near infrared absorption coefficient values. Several other candidate dielectric materials are compared in the context of achieving efficient momentum transfer from laser light.

\section{RESULTS}

The multiband electromagnetic functionality required of a relativistic light sail propelled by an intense ground-based laser beam is conceptually depicted in Fig. 1a. The light sail must simultaneously exhibit low absorption and substantial reflectivity in the propulsion band, corresponding to the Dopplerbroadened spectrum of laser wavelengths, and substantial emissivity in the thermal infrared band. This is conceptually similar to terrestrial radiative cooling, where the aim is to suppress absorption in the solar spectrum while maximizing emissivity in the 8-13 $\mu \mathrm{m}$ atmospheric transparency window. ${ }^{13-17}$ In our analysis, we assume a laser beam of wavelength $\lambda_{0}$ is perpendicularly incident on a planar light sail. The light sail has diameter $D$, but its structure is otherwise parametrized by a vector $\boldsymbol{x}$. We aim to use radiation pressure from an Earth-based laser array to accelerate the sail to a target velocity, $v_{f}$, that is some fraction of the speed of light $\beta_{f}=v_{f} / c$. If the laser power incident on the sail $\left(P_{0}\right)$ is constant during acceleration, we can approximate the distance the sail will travel $L\left(\beta_{f}\right)$ until it reaches the target velocity $\beta_{f}$ as (see Supporting Data)

$$
L\left(\beta_{f}\right) \cong\left(\frac{c^{3}}{2 P_{0}}\right) \int_{0}^{\beta_{f}} \frac{m_{s}(\boldsymbol{x})+m_{p}}{R(\boldsymbol{x}, \beta)} \frac{\gamma \beta}{(1-\beta)^{2}} \mathrm{~d} \beta,
$$


where $m_{s}(\boldsymbol{x})$ and $m_{p}$ are the mass of the sail and the payload, respectively, and $R(\boldsymbol{x}, \beta)$ is the reflectivity of the sail. In the reference frame of the light sail, the laser photons experience a Doppler shift, so we explicitly write $R(\boldsymbol{x}, \beta)=R\left(\boldsymbol{x}, \lambda^{\prime}(\beta)\right)$ where $\lambda^{\prime}(\beta)=\lambda_{0} \sqrt{(1+\beta) /(1-\beta)}$ is the red-shifted laser wavelength. Here, $\beta=v / c$ (where $v$ is velocity, and $c$ the speed of light), and $\gamma=1 / \sqrt{1-\beta^{2}}$ is the relativistic Lorentz factor. For efficient propulsion, it is desirable for the laser spot to remain focused on the area of the sail for the entire duration of the acceleration phase; that is, the diameter of the laser spot should not exceed the diameter of the sail until $\beta_{f}$ is reached. The limiting case occurs when the total acceleration distance $L\left(\beta_{f}\right)$ is equal to the diffraction-limited distance of the phased array, namely $L\left(\beta_{f}\right) \sim D d_{0} / 2 \lambda_{0}$, where $d_{0}$ is the diameter of the phased array. Substituting this back into (1), we have

$$
P_{0} d_{0}=\lambda_{0} c^{3} \int_{0}^{\beta_{f}} \frac{\rho_{S}(\boldsymbol{x}) D^{2}+m_{p}}{D} \frac{1}{R(\boldsymbol{x}, \beta)} \frac{\gamma \beta}{(1-\beta)^{2}} \mathrm{~d} \beta,
$$

where we expressed the mass of the sail as $m_{S}(\boldsymbol{x})=D^{2} \rho_{S}(\boldsymbol{x})$ with $\rho_{S}(\boldsymbol{x})$ being the area density of the sail. In practice, we seek to minimize the required phased array specifications (here given by the product of its power and size) needed to accelerate the light sail to its target velocity. As a function of the sail size $D$, expression (2) is minimized when $\rho_{S}(\boldsymbol{x}) D^{2}=m_{p}$, i.e. when the mass of the sail equals the payload mass ${ }^{7}$. Henceforth, we refer to this situation as the optimal mass (OM) condition. Under this condition, (2) further reduces to

$$
P_{0} d_{0}=2 \lambda_{0} c^{3} \sqrt{m_{p}} W(\mathbf{x}), \quad \text { where } W(\boldsymbol{x})=\int_{0}^{\beta_{f}} \frac{\sqrt{\rho_{S}(\boldsymbol{x})}}{R(\boldsymbol{x}, \beta)} \frac{\gamma \beta}{(1-\beta)^{2}} \mathrm{~d} \beta,
$$

We define $[W(\boldsymbol{x})]^{2}$ to be the reflectivity-adjusted area density (RAAD) of the structure $\boldsymbol{x}$, over the relevant Doppler-broadened spectral range. This quantity captures the tradeoff between the mass of the sail and its reflectivity, and further allows us to separate the effects of the sail structure and the payload mass. This subsequently implies that the optimal structure of the sail that minimizes $P_{0} d_{0}$ of the phased array (the product of power and size) does not depend on the payload mass.

In Fig. 1b, we visualize the relationship between payload mass, values of the RAAD $[W(\boldsymbol{x})]^{2}$, and phased array specifications, in the optimal mass condition. As mentioned, keeping in mind the constraints of atmospheric transparency, material absorption, and wavelength scaling from Eq. (3), we focus on laser array wavelengths in the near infrared (specifically, we assume $\lambda_{0}=1.2 \mu \mathrm{m}$ ). Here, and throughout this paper, $\beta_{f}=0.2$, corresponding to approximately 20 years to reach Alpha Centauri. In the context of efficient propulsion, we aim to design nanophotonic structures with the smallest RAAD, thus reducing the required power and/or size of the phased array. However, such structures must also maintain a steady-state temperature lower than a target temperature for thermal stability. The thermal balance in the light sail is achieved when the absorbed laser power (in the near infrared) equals the radiated power (in the mid-IR), namely $P_{\mathrm{abs}}(\boldsymbol{x}, \beta)=P_{\mathrm{rad}}\left(\boldsymbol{x}, T_{0}\right)$, where $T_{0}$ is the equilibrium temperature of the sail during its acceleration and

$$
P_{\mathrm{abs}}(\boldsymbol{x}, \beta)=P_{0}(\beta) A(\boldsymbol{x}, \beta),
$$




$$
P_{\text {rad }}(x, T)=D^{2} \int_{0}^{\infty} \mathrm{d} \lambda \epsilon_{\lambda}(\boldsymbol{x}, T) \pi I_{\lambda b}(T),
$$

where $P_{0}(\beta) A(\boldsymbol{x}, \beta)$ is the (Doppler-shifted) absorbed power of the sail at the near infrared wavelength $\lambda(\beta), \epsilon_{\lambda}(\boldsymbol{x}, T)$ is the (sum of the front and the back surface) hemispherical-spectral emissivity, and $I_{\lambda b}(T)$ is the blackbody spectral intensity (see Supporting Data for more details). Equating (4) and (5) in the optimal mass regime (sail mass = payload mass), we have the implicit equation for the equilibrium temperature $T_{0}$ at each velocity $\beta$ during acceleration

$$
P_{0}(\beta) A\left(\boldsymbol{x}, \beta, T_{0}\right) \rho_{S}(\boldsymbol{x})=m_{p} \int_{0}^{\infty} \mathrm{d} \lambda \epsilon_{\lambda}\left(\boldsymbol{x}, T_{0}\right) \pi I_{\lambda b}\left(T_{0}\right),
$$

Both the absorptivity in the near infrared and emissivity in the mid infrared are generally temperaturedependent quantities. In addition, we assume the light sail instantaneously reaches the equilibrium condition given by (6).

We focus on thin-film multilayer optical structures due to their ability to achieve significant optical contrast for a very low mass. A sail consisting of such a multilayer structure can be parametrized as $\boldsymbol{x}=\left(m_{1}, t_{1} ; m_{2}, t_{2} ; \ldots\right)$ where $m_{i}, t_{i}$ are the constituent material and the thickness of the $i$-th layer, respectively. Correspondingly, the area density of this structure is given by $\rho_{S}(\boldsymbol{x})=\sum_{i} \rho_{i} t_{i}$ where $\rho_{i}$ is the mass density of the $i$-th layer. We assume that the multilayer structure is uniform in the in-plane direction, and that $\boldsymbol{x}$ describes its properties in the transverse direction (along the laser beam). For each structure, the diameter $D$ is determined from the optimal mass condition, namely $D(\boldsymbol{x})=\sqrt{m_{p} / \rho_{S}(x)}$.

Silica is a natural choice for a component material of the light sail, given its very low absorption in the near infrared and substantial emissivity in the mid infrared due to vibrational resonances. In Fig. $2 b$, we show the general trends of the complex refractive index of $\mathrm{SiO}_{2}$ in the mid infrared obtained from a collection of experimental measurements ${ }^{18}$. We use the optical data from Fig. $2 b$ to model $\mathrm{SiO}_{2}$ only for the purpose of radiative cooling, i.e. Eq. (5). For the (comparatively) much narrower spectral regime relevant for propulsion and heating-Eqs. (3) and (4) - we assume the complex refractive index of the form $\tilde{n}_{\mathrm{SiO}_{2}}=n_{\mathrm{SiO}_{2}}+i \lambda \alpha_{\mathrm{SiO}_{2}} / 4 \pi$. This spectral regime is bounded by $\left[\lambda_{0}, \lambda_{f}\right]$ where $\lambda_{f} \approx 1.225 \lambda_{0}$ for $\beta_{f}=0.2$. In this analysis, we assume $\lambda_{0}=1.2 \mu \mathrm{m}$. Our motivation for employing different refractive index models stems from the vastly different magnitudes of absorption in $\mathrm{SiO}_{2}$ in the near infrared versus the mid infrared ${ }^{18}$.

We employ a nonlinear optimization to determine the thicknesses $x_{\text {opt }}$ of the $N=1,3,5,7$ layer structures that minimize the RAAD from Eq. (3), namely $W\left(\boldsymbol{x}_{\text {opt }}\right)=\min \{W(\boldsymbol{x})\}$ (see Methods). Table $\mathrm{S} 1$ contains the parameters for the respective structures, labeled A1-A7. We assume $n_{\mathrm{SiO}_{2}}=1.45$ for silica layers, and $n_{\text {gap }}=1$ (e.g. vacuum) for intermediate gap layers. Gap materials with greater-thanunity refractive index negatively impact performance due to reduced index contrast (Fig. S2). Fig. 2c shows the trend of decreasing $W$ with increasing number of layers. We observe the strongest enhancement (55\% reduction in $W$ ) is achieved from $N=1$ (A1: single $\mathrm{SiO}_{2}$ slab) to $N=3$ multi-layer structure (A3). The mechanism for enhancement comes from the fact that an additional high index $\left(\mathrm{SiO}_{2}\right)$ layer of appropriate thickness can resonantly increase reflectivity at a comparatively small cost in mass: specifically, the average reflectivity (in the $\left[\lambda_{0}, \lambda_{f}\right]$ range) of the $N=3$ structure is 0.37 , compared to 0.12 for the $N=1$ slab. Additional layers $(N=5,7)$ can further minimize $W$, though the improvements are more modest. 
Figure $2 \mathrm{~d}$ shows the equilibrium temperatures for these optimal structures, assuming a range of (near infrared) absorption coefficient values for silica. These temperatures are obtained by solving Eq. (6), for different values of the $P_{0} / m_{p}$ ratio. Varying the value of the absorption coefficient in this range does not necessitate finding different optimal structures through a repeated minimization of $W$ : for $\alpha \sim 10^{-2} \mathrm{~cm}^{-1}$ at $\lambda \sim 1.0 \mu \mathrm{m}$, the imaginary part of the refractive index is many orders of magnitude smaller than the real part, and the reflectivity (and correspondingly $W$ ) remains practically unchanged. From Fig. $2 \mathrm{~d}$, we observe that the reflectivity enhancement in structures with more layers is accompanied by stronger absorption. In contrast to the optimal thin film structures that minimize RAAD (Fig. 2c), the equilibrium temperature depends on the phased array power $P_{0}$ and the payload mass $m_{p}$ through the $P_{0} / m_{p}$ ratio in Eq. (6). Solid blue lines in Fig. 2d, show equilibrium temperatures for $P_{0} / m_{p}=$ $100 \mathrm{GW} \mathrm{g}^{-1}$ (for example, $m_{p}=1 \mathrm{~g}$, and $P_{0}=100 \mathrm{GW}$ ). For comparison, filled contours correspond to equilibrium temperature ranges for the same structures (A1-A7) for $P_{0} / m_{p}=10^{3} \mathrm{GW} \mathrm{g}^{-1}$ (red) and $P_{0} / m_{p}=10 \mathrm{GW} \mathrm{g}^{-1}$ (gray). Given that the absorption coefficient in silica glass can be lower than $10^{-5} \mathrm{~cm}^{-1}$ in the near infrared ${ }^{19}$, we conclude that silica can indeed be a promising material for thermal management of a laser-driven light sail.

An intriguing notion is to combine the radiative cooling properties of silica with a material with a larger refractive index, such as silicon. Because silica has a relatively low refractive index for its density (resulting in high RAAD), it is a suboptimal material for efficient propulsion. As the next step, we consider thin film multilayer stacks made of silica and undoped crystalline silicon (Fig. 3a). Similar to $\mathrm{SiO}_{2}$, we model the refractive index of $\mathrm{Si}$ as $\tilde{n}_{\mathrm{Si}}=n_{\mathrm{Si}}+i \lambda \alpha_{\mathrm{Si}} / 4 \pi$ in the $\left[\lambda_{0}, \lambda_{f}\right]$ spectral regime relevant for propulsion and heating. Though it has a higher refractive index, undoped silicon is not as suitable for efficient radiative cooling as silica. Due to crystalline symmetry of the silicon lattice, the fundamental vibration mode has no dipole moment and is therefore infrared inactive. As a result, the measured extinction coefficient of undoped silicon in the mid infrared is orders of magnitude smaller than that of silica $^{20,21}$, and the corresponding thin-film structures would have significantly higher steady-state temperatures (refer to Fig. S3 for comparison). For a multilayer structure that combines the highrefractive index of silicon for efficient propulsion and vibrational modes of silica for thermal management, we model the coupled analysis of propulsion and thermal stability as an inequality constraint problem, namely

$$
\begin{array}{cl}
\text { minimize } & W(\boldsymbol{x}), \\
\text { subject to } & P_{\mathrm{abs}}\left(\boldsymbol{x}, \beta_{j}\right) \leq P_{\mathrm{rad}}\left(\boldsymbol{x}, T_{s}\right), \quad \forall \beta_{j} \in\left[\beta_{0}, \beta_{f}\right]
\end{array}
$$

where we seek to find the multilayer silicon-silica structure $\boldsymbol{x}$ that minimizes RAAD, while ensuring the equilibrium temperature does not surpass some set temperature $T_{s}$. Because the reflectivity and absorptivity of the sail vary with velocity (due to Doppler shift), this condition must be true at all times during acceleration. Consequently, we must set $K$ such constraint conditions ( $1 \leq j \leq K$ in Eq. 7), one for each of the $K$ wavelengths $\lambda_{j}$ (or velocities $\beta_{j}$ ) used to discretize the $\left[\lambda_{0}, \lambda_{f}\right]$ propulsion interval. We find that $K=100$ (equivalent to $\sim 2 \mathrm{~nm}$ wavelength step) is more than sufficient to finely resolve any spectral variations in these few-layer thin-film structures.

In order to efficiently execute the joint optimization prescribed by (7), we choose to fix several parameters in the problem. In contrast to the RAAD optimization where optimal structures $x_{\text {opt }}$ only depended on the number of layers $N$ (as seen in Fig. 2c), here optimal structures also depend on the 
choice of $T_{s}, P_{0} / m_{p}, \alpha_{\mathrm{SiO}_{2}}$, and $\alpha_{\mathrm{Si}}$. In addition to $n_{\mathrm{SiO}_{2}}=1.45$ (as before), we fix $n_{\mathrm{Si}}=3.5$, and $\alpha_{\mathrm{SiO}_{2}}=10^{-6} \mathrm{~cm}^{-1}$ (chosen as a realistic value for low-loss silica). In Figure 3, we plot $W$ for optimal $N=2$ ( $\mathrm{Si}-\mathrm{SiO}_{2}$ stack), $N=3$ ( $\mathrm{Si}$-gap-SiO${ }_{2}$ stack), and $N=4$ (Si-gap-Si-SiO${ }_{2}$ stack) structures obtained from (7), for different values of $\alpha_{\mathrm{Si}}$. We assume $P_{0} / m_{p}=100 \mathrm{GW} \mathrm{g}^{-1}$ and $T_{s}=1000 \mathrm{~K}$. Furthermore, to ensure the candidate structure have feasibly realistic structures with bulk optical properties, we explicitly set a minimum layer thickness of $5 \mathrm{~nm}$ in our optimizations. For the optimal two-layer $\mathrm{Si}_{-} \mathrm{SiO}_{2}$ structure (B2 in Fig. 3a), we observe that a very thin layer of $\mathrm{SiO}_{2}$ can be enough to enable a steady-state temperature that does not exceed $1000 \mathrm{~K}$. If the silicon layer exhibits strong near infrared absorption, a thicker layer of silica is needed to maintain the same threshold temperature (B2'). However, an increase in $\mathrm{SiO}_{2}$ layer thickness negatively impacts propulsion efficiency, and $W$ correspondingly increases. We observe similar trends for optimal $N=3$ and $N=4$ silicon-silica structures. In particular, for low enough near infrared absorption coefficients, $N=4$ silicon-silica structures can have the lowest $W$ out of all analyzed structures ( $W=0.0136 \sqrt{\mathrm{g} \mathrm{m}}{ }^{-1}$ for structure B4). Nevertheless, this advantage over other analyzed structures is eliminated for stronger absorption, where a thicker $\mathrm{SiO}_{2}$ layer is necessary (B2'). As in the case of silicaonly structures (Fig. 2), most of the performance improvement relative to a single slab is realized in fewlayer structures. This is in contrast to conventional optical thin-films where many layers are usually needed to achieve the desired optical performance. In this case, additional layers come with a mass penalty, as reflected in the figure-of-merit $W(\boldsymbol{x})$. Another practical disadvantage to adding more layers is the increase in the structural and fabrication complexity.

To ensure thermal stability of the light sail, it is also important to account for the temperature dependence of absorption coefficients, which generally increase with temperature. The drawback of assuming a specific explicit form for $\alpha(T)$ is that the temperature dependence of the absorption coefficient is dependent on processing-dependent factors, such as density and type of dopants, point defects, sample preparation, etc. Instead, to maintain generality in our analysis, we assess the steady-state temperature for each structure across a wide range of assumed silicon and silica absorption coefficient values (Fig. 4). Here, we focus on a two-layer $\mathrm{Si}_{-} \mathrm{SiO}_{2}$ structure, since although silica-gap-silicon structures with additional layers can improve propulsion performance relative to silica-only structures, most of the benefit is achieved by a simple silicon-silica two-layer stack. In addition, this configuration eliminates the need for intermediate layers with near-unity refractive indices and low absorption coefficients, which might be fragile and/or difficult to fabricate. Given this, in Fig. 4, we focus on the B2' structure (sketched on the right of Fig. 4). The isotherms in Fig. 4 allow us to bound allowable $\alpha_{\mathrm{Si}_{1}}, \alpha_{\mathrm{SiO}_{2}}$ values at a given temperature, to ensure that a desired steady-state temperature can be maintained. Given a specific $\alpha(T)$ dependence (from literature or measurement), we can estimate the equilibrium temperature from Fig. 4.

Besides silicon, we also consider other potential high index materials. Figure 5 shows the minimal achievable $W$ for a single slab of optimal thickness as a function of material (near infrared) refractive index and mass density. We remark that the optimal layer thickness does not depend on material mass density, as long as a single layer structure without vacuum-like intermediate layers is used in the lightsail. We observe that typical high index dielectrics, such as $\mathrm{TiO}_{2}$ and $\mathrm{Ta}_{2} \mathrm{O}_{5}$, are advantageous compared to silica, but are considerably inferior to silicon. In contrast, molybdenum disulfide $\left(\mathrm{MoS}_{2}\right)$ could offer comparable performance due to its high refractive index in the near infrared ${ }^{22}$. As was the case with a silicon slab, any additional amount of silica would benefit the thermal stability of these high-index structures, but the amount needed depends on the target temperature and the spectrum of material 
vibrational modes, among other factors. Due to the substantial reflectivity arising from excitonic resonances, transition metal dichalcogenide (TMD) monolayers, such as $\mathrm{MoSe}_{2}{ }^{23,24}$ could also be of interest, provided sufficient reflectivity can be realized over the relevant Doppler-broadened spectral range.

\section{DISCUSSION}

Our work shows that optimized thin film structures could be promising candidates for a laserdriven light sail, providing the means of efficient propulsion as well as radiative cooling. For the optimal mass case (sail mass = payload mass), we introduced the concept of reflectivity adjusted area density (RAAD) as one relevant figure of merit that seeks to minimize the constraints on the power and the size of the laser array. In addition, we demonstrated that structures which combine materials with high mid infrared extinction coefficients (such as $\mathrm{SiO}_{2}$ ) and high near infrared refractive index (such as $\mathrm{Si}$ ) can be tailored to maintain a desired steady-state temperature for a range of near infrared absorption coefficient values. The structures and materials analyzed in this study are intended to highlight the versatility of multilayer thin film structures to achieve a desired multiband optical response for a very low mass. In addition to materials analyzed here, other potential candidates could include IR-active materials such as silicon nitride, silicon carbide, or doped silicon, to name a few.

Furthermore, it will be important to maintain the mechanical stability of the light sail under high power illumination and acceleration, which was not addressed in this paper. As our analysis shows, efficient propulsion and radiative cooling could in principle be achieved in very thin $(\sim 100 \mathrm{~nm})$ structures. Fabrication, assembly, and handling of high quality, ultra-thin films across large $\left(\sim \mathrm{m}^{2}\right)$ areas presents an additional engineering challenge ${ }^{10}$. Porous, low density materials, such as aerogels ${ }^{25-28}$, may provide additional structural stability and present an opportunity for future research and investigation. Finally, the results presented here highlight the relevant scaling and parameter dependencies, and are equally applicable to relativistic interstellar light sailing as well as other regimes of laser-driven propulsion.

\section{ACKNOWLEDGEMENTS}

This work was supported by the Caltech Space Solar Power project (CMW) and by the DOE 'LightMaterial Interactions in Energy Conversion' Energy Frontier Research Center under grant DESC0001293(OI). CMW acknowledges fellowship support from the Resnick Sustainability Institute and from an NSF Graduate Research Fellowship (grant number 1745301). We acknowledge helpful discussions with Zac Manchester, Mike Kelzenberg, Michelle Sherrott, Artur Davoyan, Deep Jariwala, William Whitney, Joeson Wong, and Kevin Parkin.

\section{METHODS}

Optical properties (reflection, transmission, absorption) of heterostructure stacks analyzed in this work are obtained via the transfer matrix method ${ }^{29}$. Material properties are drawn from referenced literature data ${ }^{18,20,21}$. For unconstrained minimization of the reflectivity-adjusted area density $[W(\boldsymbol{x})]^{2}$ from Eq. (3), we find the optimal heterostructure $\boldsymbol{x}_{\mathrm{opt}}$ via the limited memory Broyden-FletcherGoldfarm-Shanno (BFGS) algorithm ${ }^{30}$. For constrained minimization (Eq. 7) that ensures the equilibrium temperature does not surpass a set target temperature $T_{s}$, we use the method-of-moving-asymptotes (MMA) algorithm ${ }^{31}$. Both algorithms are accessed via the NLopt package ${ }^{32}$. Because these are local optimization algorithms, we initialize $\sim 2000$ different starting points $x$ (with thicknesses in the relevant 
range) for each desired number of layers and material arrangement to arrive at the final structures reported in Table S1.

\section{SUPPORTING INFORMATION AVAILABLE}

Parameters for analyzed heterostructures (Table S1); expressions for propulsion and radiative cooling of a laser-driven lightsail; sensitivity of figure of merit to gap refractive index; infrared extinction coefficients of silica \& silicon.

\section{REFERENCES}

(1) Urbanczyk, M. G. R. Solar Sails - A Realistic Propulsion for Spacecraft; 1967.

(2) McInnes, C. R. Solar Sailing: Technology, Dynamics and Mission Applications; Springer Praxis Books; Springer London, 2013.

(3) Tsuda, Y.; Mori, O.; Funase, R.; Sawada, H.; Yamamoto, T.; Saiki, T.; Endo, T.; Kawaguchi, J. Flight Status of IKAROS Deep Space Solar Sail Demonstrator. Acta Astronaut. 2011, 69 (9-10), 833-840.

(4) Marx, G. Interstellar Vehicle Propelled By Terrestrial Laser Beam. Nature 1966, 211 (5044), $22-$ 23.

(5) Redding, J. L. Interstellar Vehicle Propelled by Terrestrial Laser Beam. Nature 1967, 213 (5076), 588-589.

(6) Forward, R. L. Roundtrip Interstellar Travel Using Laser-Pushed Lightsails. J. Spacecr. Rockets 1984, 21 (2), 187-195.

(7) Lubin, P. A Roadmap to Interstellar Flight. JBIS 2016, 69, 40-72.

(8) Breakthrough Starshot https://breakthroughinitiatives.org/Initiative/3.

(9) Landis, G. A. Optics and Materials Considerations for a Laser-Propelled Lightsail. 40th Congr. Int. Astronaut. Fed. 1989, No. IAA-89-664.

(10) Atwater, H. A.; Davoyan, A. R.; Ilic, O.; Jariwala, D.; Sherrott, M. C.; Went, C. M.; Whitney, W. S.; Wong, J. Materials Challenges for the Starshot Lightsail. 2017.

(11) Bible, J.; Johansson, I.; Hughes, G. B.; Lubin, P. M. Relativistic Propulsion Using Directed Energy. Nanophotonics Macrophotonics Sp. Environ. VII 2013.

(12) Kulkarni, N.; Lubin, P.; Zhang, Q. Relativistic Spacecraft Propelled by Directed Energy. Astron. J. 2018, 155 (4), 155.

(13) Catalanotti, S.; Cuomo, V.; Piro, G.; Ruggi, D.; Silvestrini, V.; Troise, G. The Radiative Cooling of Selective Surfaces. Sol. Energy 1975, 17 (2), 83-89.

(14) Granqvist, C. G.; Hjortsberg, A. Radiative Cooling to Low Temperatures: General Considerations and Application to Selectively Emitting SiO Films. J. Appl. Phys. 1981, 52 (6), 4205-4220.

(15) Gentle, A. R.; Smith, G. B. Radiative Heat Pumping from the Earth Using Surface Phonon Resonant Nanoparticles. Nano Lett. 2010, 10 (2), 373-379.

(16) Raman, A. P.; Anoma, M. A.; Zhu, L.; Rephaeli, E.; Fan, S. Passive Radiative Cooling below Ambient Air Temperature under Direct Sunlight. Nature 2014, 515 (7528), 540-544.

(17) Zhai, Y.; Ma, Y.; David, S. N.; Zhao, D.; Lou, R.; Tan, G.; Yang, R.; Yin, X. ScalableManufactured Randomized Glass-Polymer Hybrid Metamaterial for Daytime Radiative Cooling. Science (80-. ). 2017, 355 (6329), 1062-1066.

(18) Kitamura, R.; Pilon, L.; Jonasz, M. Optical Constants of Silica Glass from Extreme Ultraviolet to Far Infrared at near Room Temperature. Appl. Opt. 2007, 46 (33), 8118.

(19) Chigusa, Y.; Yamamoto, Y.; Yokokawa, T.; Sasaki, T.; Taru, T.; Hirano, M.; Kakui, M.; Onishi, M.; Sasaoka, E. Low-Loss Pure-Silica-Core Fibers and Their Possible Impact on Transmission Systems. J. Light. Technol. 2005, 23 (11), 3541-3550. 
(20) Palik, E. D. Handbook of Optical Constants of Solids; Palik, E. D., Ed.; Elsevier, 1998.

(21) Chandler-Horowitz, D.; Amirtharaj, P. M. High-Accuracy, Midinfrared Refractive Index Values of Silicon. J. Appl. Phys. 2005, 97 (12), 123526.

(22) Roxlo, C. B.; Chianelli, R. R.; Deckman, H. W.; Ruppert, A. F.; Wong, P. P. Bulk and Surface Optical Absorption in Molybdenum Disulfide. J. Vac. Sci. Technol. A Vacuum, Surfaces, Film. 1987, 5 (4), 555-557.

(23) Scuri, G.; Zhou, Y.; High, A. A.; Wild, D. S.; Shu, C.; De Greve, K.; Jauregui, L. A.; Taniguchi, T.; Watanabe, K.; Kim, P.; et al. Large Excitonic Reflectivity of Monolayer MoSe2 Encapsulated in Hexagonal Boron Nitride. Phys. Rev. Lett. 2018, 120 (3).

(24) Back, P.; Zeytinoglu, S.; Ijaz, A.; Kroner, M.; Imamoglu, A. Realization of an Electrically Tunable Narrow-Bandwidth Atomically Thin Mirror Using Monolayer MoSe2. Phys. Rev. Lett. 2018, $120(3)$.

(25) Pierre, A. C.; Pajonk, G. M. Chemistry of Aerogels and Their Applications. Chem. Rev. 2002, 102 (11), 4243-4266.

(26) Jo, M.-H.; Hong, J.-K.; Park, H.-H.; Kim, J.-J.; Hyun, S.-H.; Choi, S.-Y. Application of SiO2 Aerogel Film with Low Dielectric Constant to Intermetal Dielectrics. Thin Solid Films 1997, 308 309, 490-494.

(27) Leventis, N.; Sotiriou-Leventis, C.; Zhang, G.; Rawashdeh, A.-M. M. Nanoengineering Strong Silica Aerogels. Nano Lett. 2002, 2 (9), 957-960.

(28) Zu, G.; Shen, J.; Zou, L.; Wang, W.; Lian, Y.; Zhang, Z.; Du, A. Nanoengineering Super HeatResistant, Strong Alumina Aerogels. Chem. Mater. 2013, 25 (23), 4757-4764.

(29) Macleod, H. A. Thin-Film Optical Filters, 4th ed.; CRC Press / Taylor and Francis Group, LLC, Boca Raton, FL, 2010.

(30) Nocedal, J. Updating Quasi-Newton Matrices with Limited Storage. Math. Comput. 1980, 35 (151), 773.

(31) Svanberg, K. A Class of Globally Convergent Optimization Methods Based on Conservative Convex Separable Approximations. SIAM J. Optim. 2002, 12 (2), 555-573.

(32) Johnson, S. G. The NLopt nonlinear-optimization package http://ab-initio.mit.edu/nlopt. 

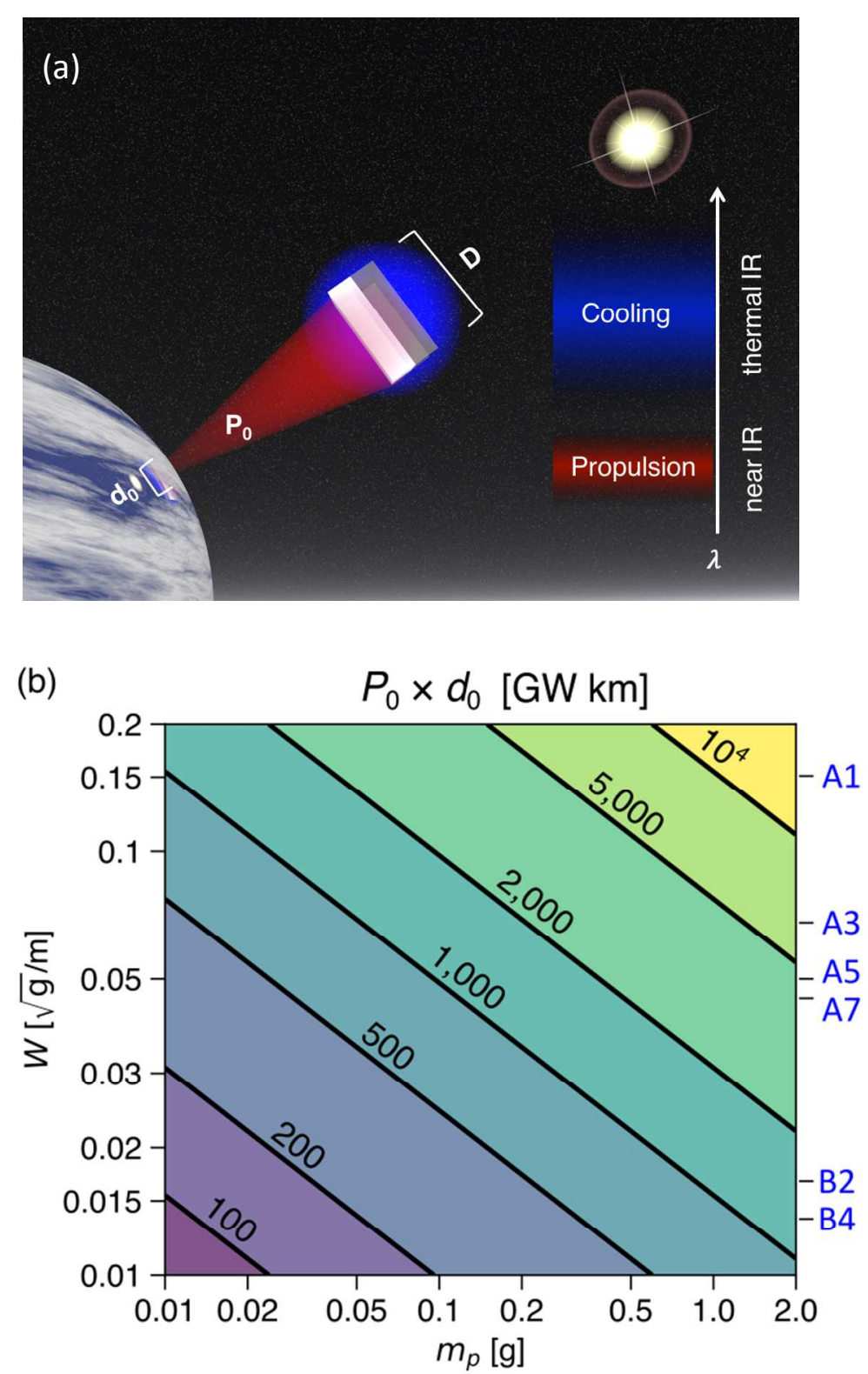

Fig 1. (a) Operating principle for propulsion and thermal management of a cosmic nanophotonic light sail. Radiation pressure from an Earth-based phased array (of size $d_{0}$ and power $P_{0}$ ) propels the sail (of size $D$ ). For the light sail, multiband functionality is needed: low absorptivity in the (Doppler-broadened) spectral regime corresponding to the incident laser (red), and high emissivity in mid-IR for radiative cooling (blue). Given the constraints of atmospheric transparency, material absorption, and wavelength scaling, we focus on propulsion wavelengths in the near infrared. (b) Phased array specifications, power $\times$ size $\left(P_{0} \times d_{0}\right)$, necessary to accelerate the nanophotonic light sail to $0.2 c$ for a given $m_{p}$ (payload mass) and $W$ (where $W^{2}$ is the reflectivity-adjusted area density from Eq. (3)). Right: corresponding $W$ values for structures analyzed in this work, listed in Table S1. 
(a)

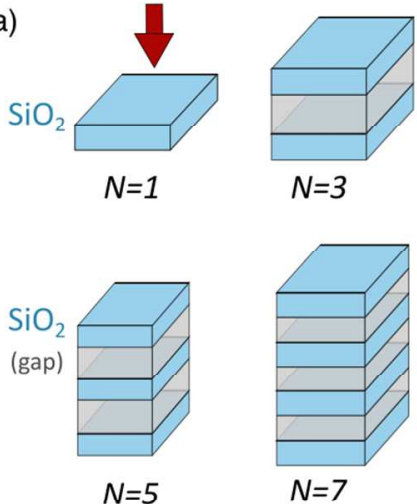

(c)

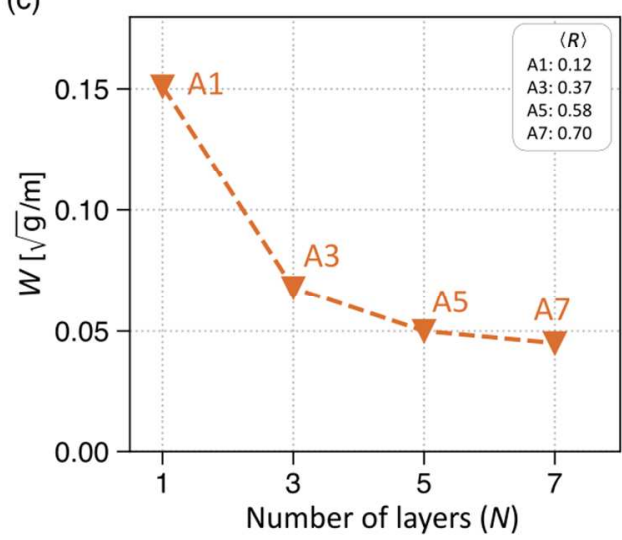

(b)

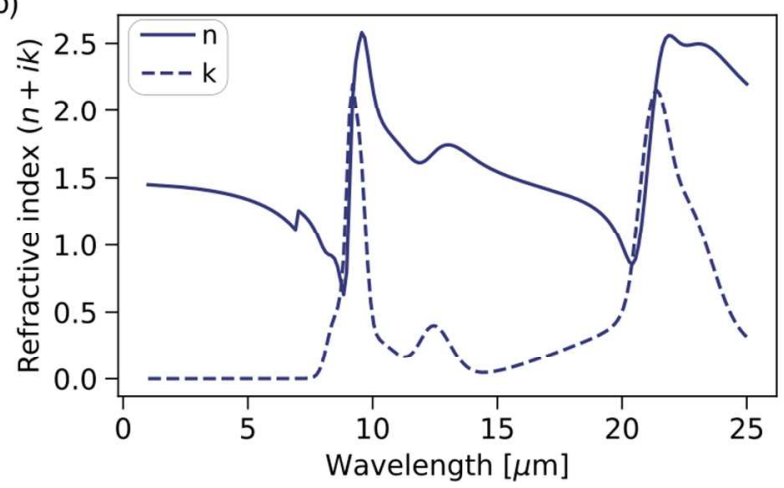

(d)

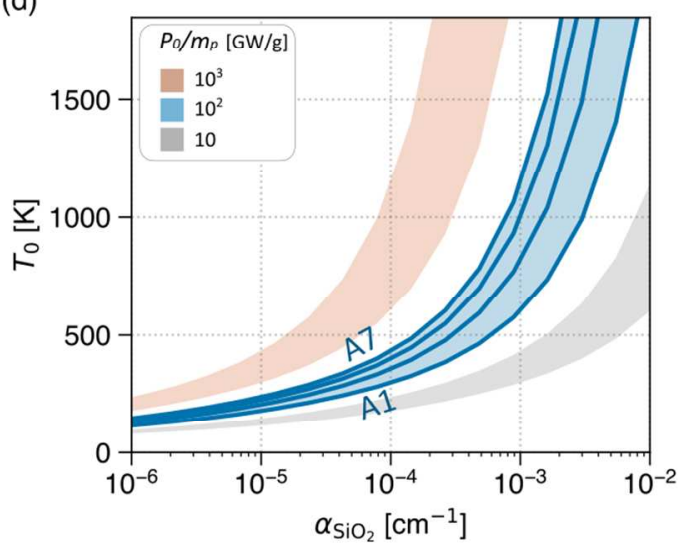

Fig 2. (a) Light sail made from thin-film stacks of $\mathrm{SiO}_{2}$ multilayers (separated by a gap of unity ref. index). Red arrow denotes the incident laser light. (b) Complex refractive index of $\mathrm{SiO}_{2}$ in the mid-IR used to model radiative cooling. (c) Optimal $N=1,3,5,7$ multilayer structures (labeled A1-7) that minimize $W^{2}$, the reflectivity-adjusted area density (RAAD) from Eq. (3). Inset shows mean reflectance in the laser band. Structure parameters are given in Table S1. (d) Equilibrium temperature $T_{0}$ of the same structures from (c), for different values of the near-IR absorption coefficient. Reflectivity enhancement in structures with more layers is accompanied by stronger absorption. The temperature depends on the $P_{0} / m_{p}$ ratio (Eq. 6). 

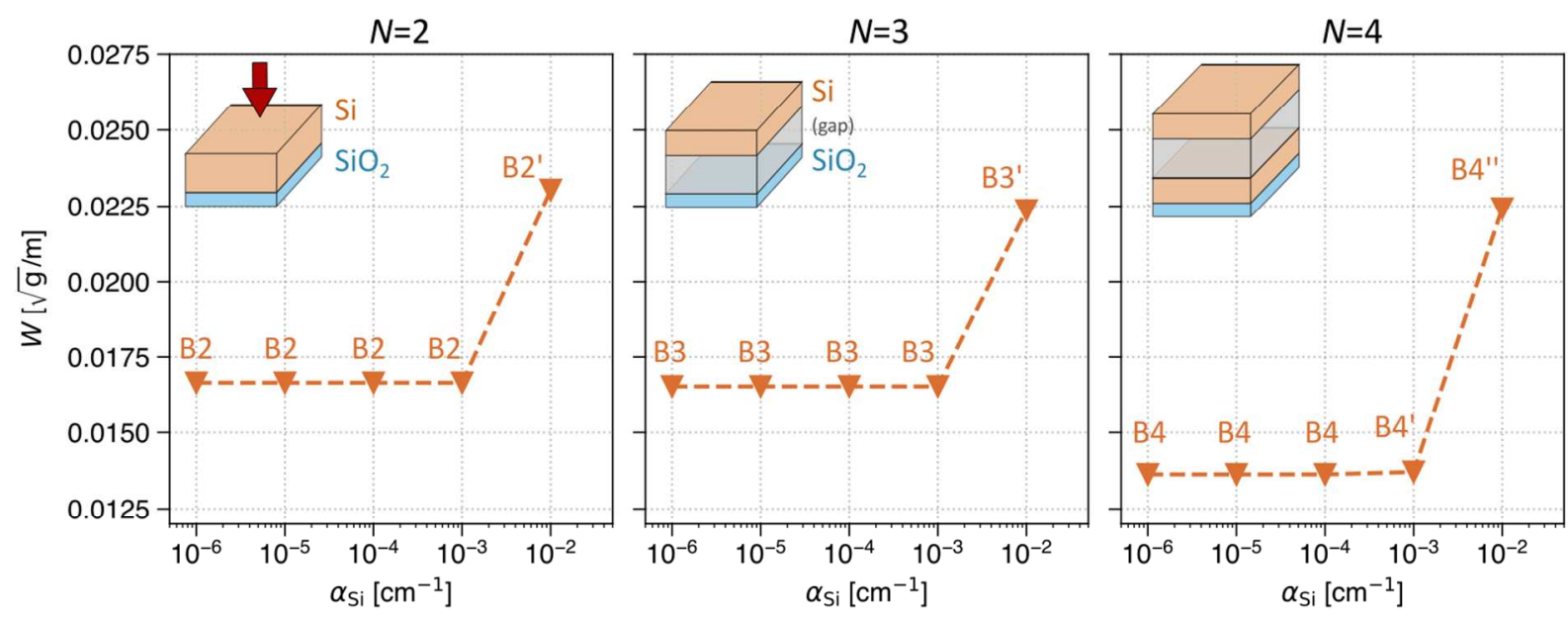

Fig 3. Thin-film structures with $\mathrm{Si}$ and $\mathrm{SiO}_{2}$ layers that minimize the reflectivity-adjusted area density $W^{2}$ through the constrained optimization defined in Eq. (7), for different values of $\alpha_{S \mathrm{i}}$. Here, $P_{0} / m_{p}=100 \mathrm{GW} \mathrm{g}^{-1}$ and the maximum allowed temperature is set at $T_{s}=1000 \mathrm{~K}$. Structure parameters are given in Table S1. Higher refractive index of silicon combined with radiative cooling properties of silica enables much more efficient propulsion relative to silica-only structures from Fig. 2. For large absorption (e.g. $\alpha=10^{-2} \mathrm{~cm}^{-1}$ ), thicker layers of silica are needed to ensure equilibrium sail temperature does not surpass $T_{s}$; as a result, there is a penalty in $W$. Red arrow denotes the incident laser light. 


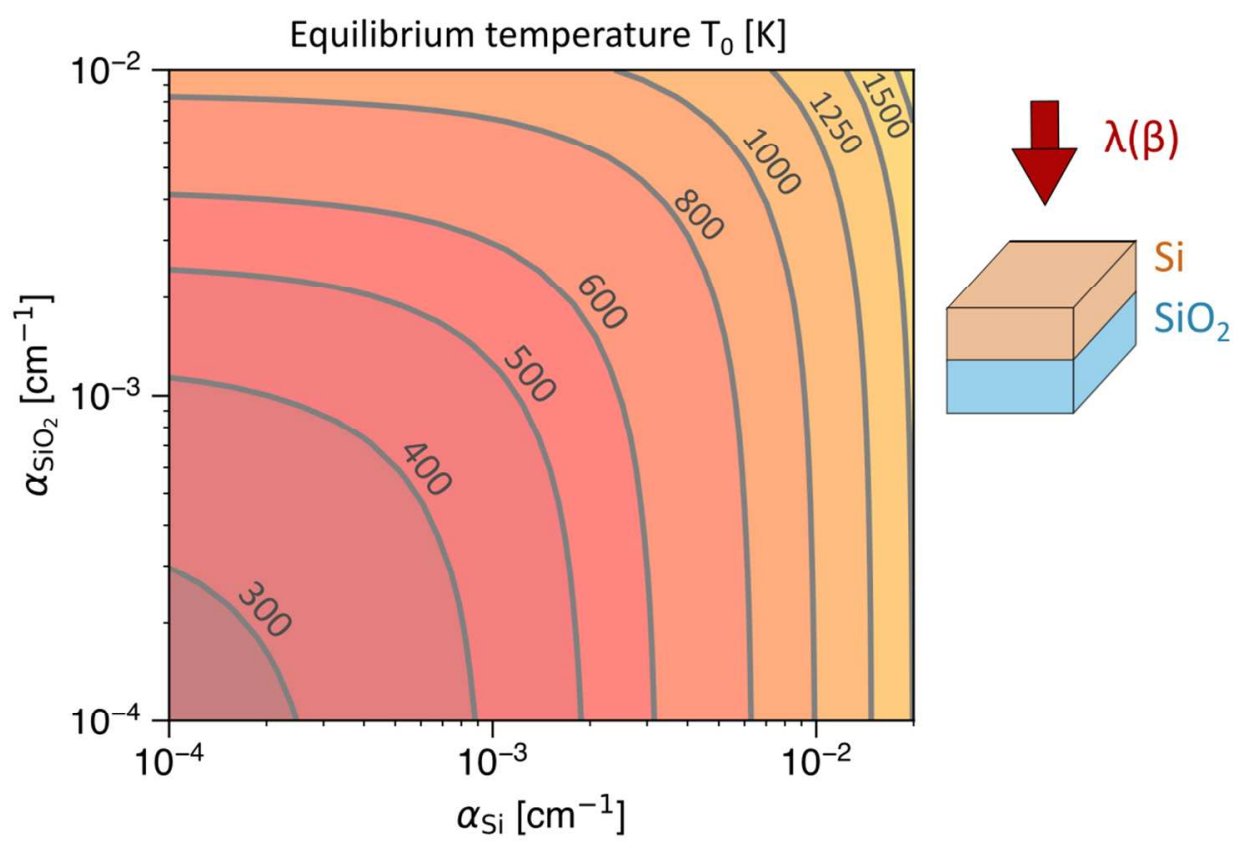

Fig 4. Equilibrium temperature for the $\mathrm{Si}_{-} \mathrm{SiO}_{2}$ two-layer structure $\mathrm{B} 2$ ' from Fig. 3, for different values of the $\mathrm{Si}$ and $\mathrm{SiO}_{2}$ absorption coefficients. For this structure, $W=$ $0.023[\sqrt{\mathrm{g}} / \mathrm{m}]$, and the layer thicknesses are listed in Table S1. Here we assume $P_{0} / m_{p}=$ $100 \mathrm{GW} \mathrm{g}^{-1}$. 


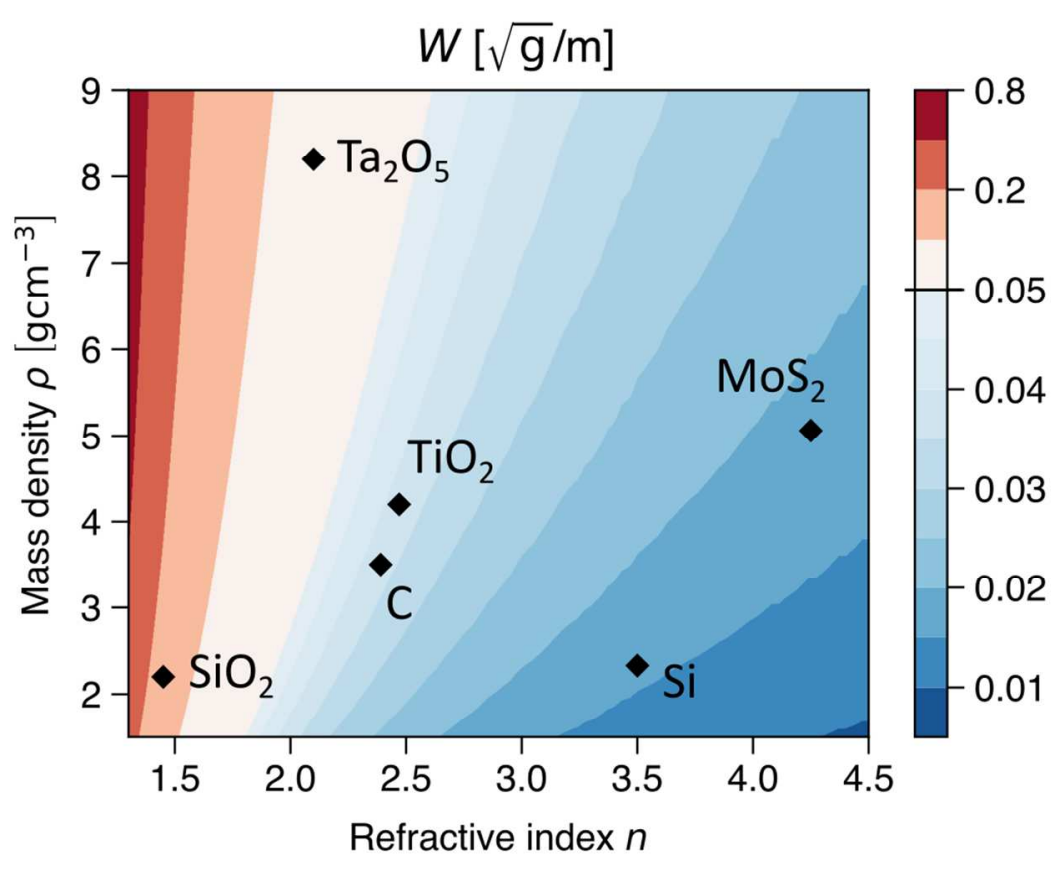

Fig 5. Minimal achievable $W$ for a single slab of optimal thickness, as a function of material refractive index and mass density. Note the scale change at $W=0.05 \sqrt{\mathrm{g}} \mathrm{m}^{-1}$. For reference, the $\mathrm{SiO}_{2}$ structure corresponds to the structure A1 from Fig. 2. The Si structure here is similar, but not equivalent, to structure $\mathrm{B} 2$ from Fig. 3 (B2 also includes a $5 \mathrm{~nm}$ layer of $\mathrm{SiO}_{2}$, for cooling). 


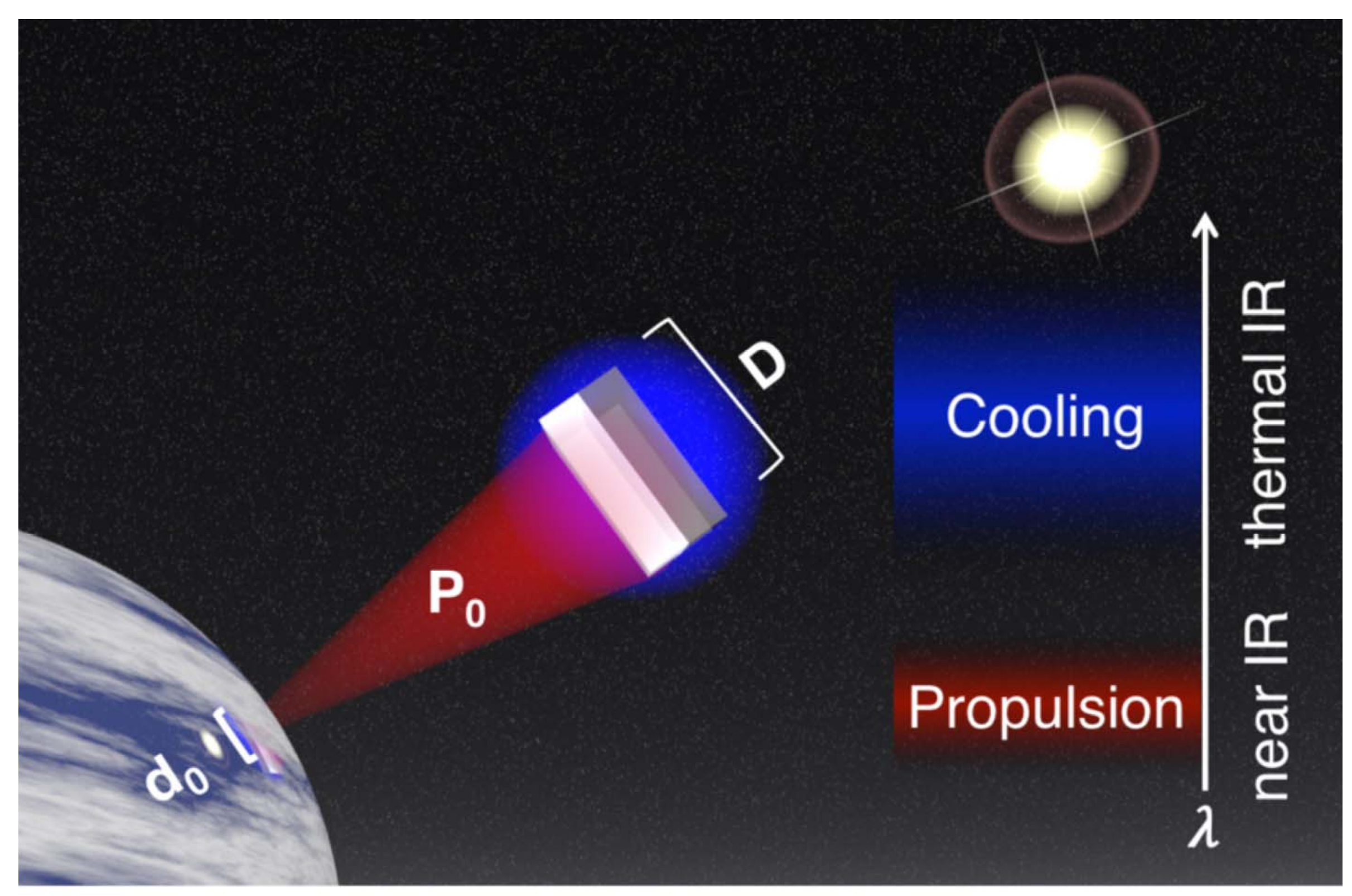

